\title{
Health-related quality of life in patients with congenitally corrected transposition of the great arteries
}

\author{
Michael G. Gaies, MD, MPH, ${ }^{\mathrm{a}}$ Caroline S. Watnick, BS, ${ }^{\mathrm{a}}$ James G. Gurney, PhD, ${ }^{\mathrm{a}}$ Edward L. Bove, MD, \\ and Caren S. Goldberg, MD, MS
}

\begin{abstract}
Objective: The study's objectives were to survey the quality of life in patients with congenitally corrected transposition of the great arteries and to compare the responses of those who have undergone anatomic repair with those who have a systemic right ventricle.
\end{abstract}

\begin{abstract}
Methods: Thirty-eight patients who underwent anatomic repair and 13 patients after either conventional repair or no surgical procedure were enrolled. Subjects completed the PedsQL 4.0 Generic and 3.0 Cardiac Modules. Scores were also compared with those of patients from the literature with at least moderately severe cardiovascular disease. Mean differences between groups were compared, and the association between clinical variables and score in the anatomic repair subgroup was measured.
\end{abstract}

Results: Caregivers of patients in the anatomic repair group reported similar scores compared with the nonanatomic repair group in all functional domains The anatomic repair group self-reported lower school function (63 vs $81, P=.02$ ). On the Cardiac Module, patients in the anatomic repair group self-reported fewer problems related to residual heart disease ( 75 vs 63 ), appearance ( 81 vs 68 ), and treatment anxiety ( 74 vs 59 ), although the differences were not significant. Compared with patients with other heart disease, the anatomic repair group scored lower, with the largest differences in cognition and communication. Prolonged hospital stay and need for a pacemaker were associated with lower quality of life after anatomic repair.

Conclusions: Patients in the anatomic repair group had similar quality of life compared with patients in the nonanatomic group, except in the domain of school functioning. Prolonged hospital stay and need for a pacemaker after anatomic repair may be risk factors for lower quality of life. (J Thorac Cardiovasc Surg 2011;142:136-41)

Anatomic surgical repair of congenitally corrected transposition of the great arteries (CCTGA) involves restoration of the morphologic left ventricle to the systemic circulation using an atrial switch (Senning or Mustard procedure) in conjunction with an arterial switch or a Rastelli procedure. These complex operations have become feasible for patients with CCTGA with low rates of morbidity and mortality reported. ${ }^{1-7}$ Supporters of anatomic repair (AR) do so in part because of concerns about the long-term function of the morphologic right ventricle in the systemic circulation. Graham and colleagues ${ }^{8}$ demonstrated that moderate or severe ventricular dysfunction with or without clinical heart failure will develop by the fifth decade in the majority of patients with CCTGA and a systemic right ventricle, and that $30 \%$ to $40 \%$ report functional limitations. Many institutions have adopted AR as the preferred approach for suit-

\footnotetext{
From the Division of Cardiology, ${ }^{\mathrm{a}}$ Department of Pediatrics and Communicable Diseases, and Division of Pediatric Cardiac Surgery, ${ }^{b}$ Department of Surgery, University of Michigan School of Medicine, Ann Arbor, Mich.

Disclosures: Authors have nothing to disclose with regard to commercial support.

Received for publication July 21, 2010; revisions received Sept 16, 2010; accepted for publication Nov 25, 2010; available ahead of print Jan 31, 2011

Address for reprints: Michael G. Gaies, MD, MPH, CS Mott Children's Hospital, L1242 Women's, SPC 5204, 1500 East Medical Center Drive, Ann Arbor, MI 48109-5204 (E-mail: mgaies@med.umich.edu).

$0022-5223 / \$ 36.00$

Copyright (c) 2011 by The American Association for Thoracic Surgery doi:10.1016/j.jtcvs.2010.11.035
}

able patients with the intent to improve these outcomes. However, the greater risk of early morbidity and mortality after AR compared with conventional repair and the lack of data on long-term outcomes after AR spark debate about the optimal surgical strategy for patients with CCTGA.

Perceived health-related quality of life (QOL) is an increasingly important consideration in patients with chronic medical conditions. QOL has been assessed in several populations of patients with chronic disease, ${ }^{9}$ and more recently in patients with congenital heart disease (CHD) ${ }^{10,11}$ Uzark and colleagues ${ }^{10}$ validated a tool for the assessment of QOL in patients with CHD, the Pediatric Quality of Life Inventory (Peds QL) and the related Peds QL Cardiac Module, and have demonstrated its utility in studying children with varying degrees of CHD severity. ${ }^{11}$

Because the primary theoretic advantage of $\mathrm{AR}$ is to improve long-term functional status, we believe QOL is an important outcome in the calculus of relative risks and benefits of this strategy. We undertook this study to compare the 2 management strategies using the Peds QL and Peds QL Cardiac Modules. We measured the perceived QOL of anatomically repaired patients at intermediate follow-up and compared their responses with those of patients who had undergone conventional or no repair. Our secondary aims were to (1) compare the QOL outcomes for patients with CCTGA with those of patients with other forms of 


\section{Abbreviations and Acronyms \\ $\mathrm{AR}=$ anatomic repair \\ CCTGA $=$ congenitally corrected transposition of the great arteries \\ CHD $=$ congenital heart disease \\ NAR $=$ non-anatomic repair \\ Peds QL $=$ Pediatric Quality of Life Inventory \\ $\mathrm{QOL}=$ quality of life}

CHD and (2) determine the association between clinical risk factors and perceived QOL for patients who have undergone AR.

\section{MATERIALS AND METHODS \\ Subjects}

All patients from our institution born after 1983 with atrioventricular and ventriculoarterial discordance and 2 functional ventricles had been previously identified for a retrospective review of surgical outcomes after AR. ${ }^{12}$ Patients who were treated medically or who had undergone conventional repair were identified from our institution's database. AR consisted of a Senning procedure plus an arterial switch operation in patients with suitable pulmonary valves or a Rastelli procedure in patients with valvar pulmonary stenosis/atresia or complex left ventricular outflow tract obstruction. Several patients in the AR group were palliated at other institutions before receiving their definitive procedure at our center. The indications for referral for AR were mixed. In some cases AR was the first option, whereas others were referred after failed attempts at conventional or single-ventricle repair.

After receiving approval from our institutional review board, known survivors after AR (45) and other patients with CCTGA (18) were contacted by mail in June 2008. Participants had to be at least 6 months removed from their most recent surgical procedure to be eligible for this study, consistent with the criteria used to validate the Peds QL. ${ }^{11}$ If there was no response, phone calls were made on a weekly basis up to 3 times. Written consent was obtained for each subject. Participating families were asked to complete the age-appropriate version of the PedsQL 4.0 Generic Core Scale and 3.0 Cardiac Module, and they received a small gift as a token of appreciation.

\section{Measures}

The Peds QL is a multi-item questionnaire assessing perceived healthrelated QOL across a wide age range (2-18 years). It can be completed within 5 to 10 minutes. The 23-item Peds QL 4.0 Generic Core Scales assess physical ( 8 items), emotional (5 items), social (5 items), and school functioning ( 5 items). For ages 2 to 4 years, only parental proxy response is offered, but separate self-report and parent-proxy forms exist for ages 5 to 7,8 to 12 , and 13-18 years. A young adult self-report form is available for patients aged 18 to 25 years. As suggested by Uzark and colleagues ${ }^{11}$ families of patients aged 7 years or less completed the parent-proxy questionnaire only; families of patients between the ages of 8 and 17 years were also given the self-report form. Patients aged 18 years or more were given the self-report form only.

The 3.0 Cardiac Module also has 5 scales assessing QOL related to residual cardiac symptoms ( 7 items), physical appearance ( 3 items), treatment anxiety (4 items), cognition (5 items), and communication (3 items). Additional items are embedded to assess for problems related to medication adherence. The validity and reliability of the Cardiac Module have been demonstrated. ${ }^{10}$
TABLE 1. Patient demographics

\begin{tabular}{lcc}
\hline \multicolumn{1}{c}{ Characteristic } & $\begin{array}{c}\text { Anatomic } \\
\text { Repair }\end{array}$ & $\begin{array}{c}\text { Non-anatomic } \\
\text { repair }\end{array}$ \\
\hline Response rate, n $(\%)$ & $38(84 \%)$ & $13(72 \%)$ \\
Age, $\mathrm{y}($ mean $\pm \mathrm{SD})$ & $7.26 \pm 4.34$ & $14.31 \pm 4.53$ \\
$2-4, \mathrm{n}(\%)$ & $13(34 \%)$ & $0(0 \%)$ \\
$5-7$ & $10(26 \%)$ & $1(8 \%)$ \\
$8-12$ & $10(26 \%)$ & $3(23 \%)$ \\
$13-18$ & $4(11 \%)$ & $7(54 \%)$ \\
$19-25$ & $1(3 \%)$ & $2(15 \%)$ \\
Gender, $\mathrm{n}(\%)$ & $13(34 \%)$ & $9(69 \%)$ \\
Female & & $11(85 \%)$ \\
Forms completed, $\mathrm{n}(\%)$ & $37(97 \%)$ & $11(85 \%)$ \\
Parent-proxy form & $13(34 \%)$ & $9(69 \%)$ \\
Self-report form & $12(32 \%)$ & $\mathrm{N} / \mathrm{A}$ \\
Both forms & 68 & \\
Duration since AR, mo (mean) &
\end{tabular}

$A R$, Anatomic repair; $N / A$, not applicable; $S D$, standard deviation.

All items use a 5-point Likert scale designed to assess QOL and level of functioning. Respondents report how often they experience specific problems grouped by functional domain ( 0 , never a problem; 1 , almost never; 2 , sometimes; 3 , often; 4, almost always a problem). Items are reverse-scored and linearly transformed to a scale from 0 to 100 , with 100 representing no problems within a functional domain and the highest possible QOL score. The emotional, social, and school functioning scores can be combined and averaged to report a psychosocial health summary score. A general population will report average scores in individual domains ranging from 77 to 89 . $^{9}$

\section{Outcomes and Statistical Analyses}

Scores for total QOL and within each individual functional domain were tabulated using the linearly transformed method described. Perceived health-related QOL of patients in the AR group was compared with that of patients who had undergone conventional or non-anatomic repair (NAR) in each functional domain using Student $t$ tests.

To compare QOL outcomes for the AR group with a cohort of those with moderate to severe $\mathrm{CHD}$, a published control group was selected from the study by Uzark and colleagues ${ }^{11}$ judged to have "moderate cardiovascular disease requiring no therapy or surgically corrected (curative), or surgically corrected cardiovascular disease with significant residua or need for additional surgery." This combined group was selected to approximate what might be expected for patients with CCTGA and a systemic right ventricle in the modern era, and thus serves as a reasonable benchmark for NAR strategies. The scores for this combined group for each scale were calculated from weighted averages of these 2 samples. Graphic analysis and 2-sample $t$ tests were performed comparing the AR group with this group.

Finally, we analyzed clinical risk factors for poor QOL after AR. We identified 3 risk factors a priori that we hypothesized could be associated with perceived QOL: (1) major in-hospital morbidity (at least 1 of the following: mechanical circulatory support, dialysis, or documented neurologic injury as defined by stroke or seizures); (2) prolonged hospital stay; and (3) pacemaker-dependence. Hospital length of stay was dichotomized to prolonged ( $\geq 28$ days) or not ( $<28$ days). Student $t$ test was used to compare outcomes between those with and without each clinical factor.

\section{RESULTS}

Questionnaires were received from 38 subjects in the AR group $(84 \%)$ and 13 subjects in the NAR group $(72 \%)$, for an enrollment rate of $81 \%(51 / 63)$. Patient demographics are shown in Table 1. Subjects in the AR group were 
generally younger. Twenty-six $(68 \%)$ of the patients in the AR group underwent a Senning/arterial switch procedure, and 12 patients $(32 \%)$ underwent a Senning/Rastelli procedure. Seven of the 38 respondents $(18 \%)$ had required surgical or catheter-based reintervention. Nine of the 38 respondents $(24 \%)$ were pacemaker-dependent; 6 of 9 respondents $(67 \%)$ had a pacemaker before AR. In the NAR group, 11 patients had undergone conventional surgical repair: 4 had left ventricle to pulmonary artery conduits placed, 4 had ventricular septal defect closure as the primary procedure, and 3 had tricuspid valve repair or replacement. Of these 11 patients who underwent conventional surgical repair, 3 had a single procedure and the other 8 had 2 or more procedures. Three of the 13 patients $(23 \%)$ in the NAR group were pacemaker-dependent.

\section{Comparing Quality of Life in Anatomic Repair Versus Non-Anatomic Repair Groups}

The QOL scores for the AR and NAR groups on both the Generic Core Scales and the Cardiac Module are displayed in Figure 1. On the Generic Scales, parent-proxies of the AR group tended to report similar or higher QOL scores in each functional domain, with no statistically significant differences between the groups. The AR group tended to selfreport better physical functioning than the NAR group, but reported a significantly lower school functioning score (AR 63 vs NAR 81, $P=.02$ ). The AR group self-reported

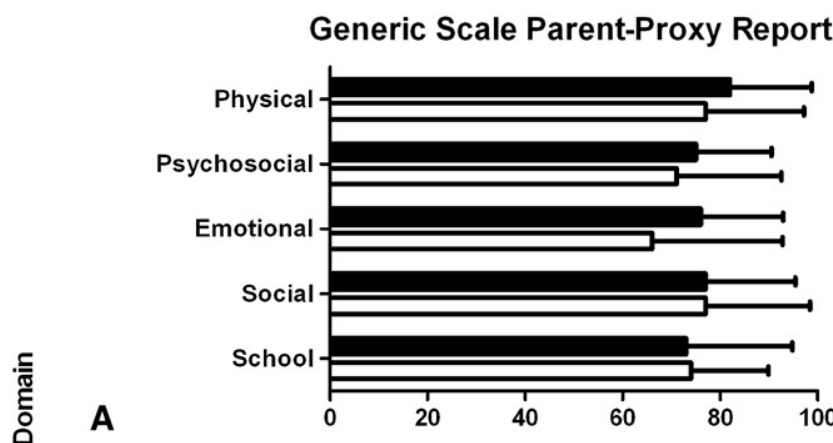

Cardiac Module Parent-Proxy

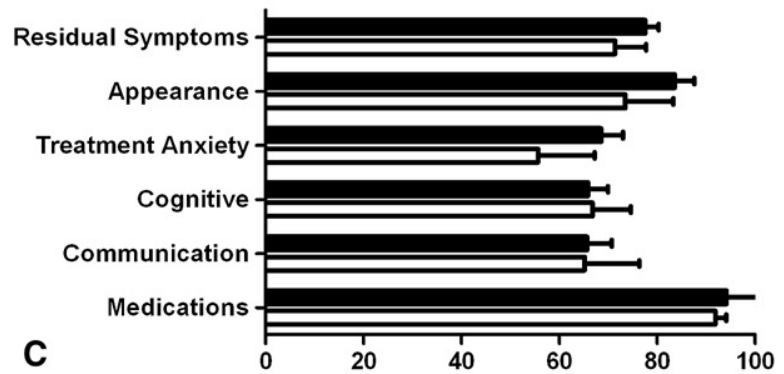

lower scores in each component of the psychosocial health domain (emotional, social, and school functioning) compared with parent-proxy reporting, whereas the NAR group self-reported similar scores to parents.

On the Cardiac Module, the AR group tended to report better scores compared with the NAR group related to symptoms, physical appearance, and treatment anxiety on both forms. Both groups reported low scores in the domains of cognition and communication relative to the general population. The AR group's self-reported cognitive functioning was lower than the NAR group's, although this was not statistically significant (AR 55 vs NAR $68, P=.3$ ).

\section{Comparing Quality of Life in the Anatomic Repair Group Versus Patients With Other Forms of Moderate-Severe Cardiac Disease}

The scores of both the AR group and the cohort of patients with other forms of moderate-severe CHD are shown in Figure 2. On the Cardiac Module, the AR group's scores in the domains of symptoms and physical appearance were similar by both parent-proxy and self-report to those of patients in the mixed cohort. There was a consistent pattern on both forms for lower reported QOL by the AR group regarding treatment anxiety, cognition, and communication. There were greater discrepancies in the parent-proxy reported domains of cognitive function (AR 55 vs other $77, P=.03$ ) and communication (AR 66 vs other 76, $P=.06$ ).

\section{Generic Scale Self-Report}

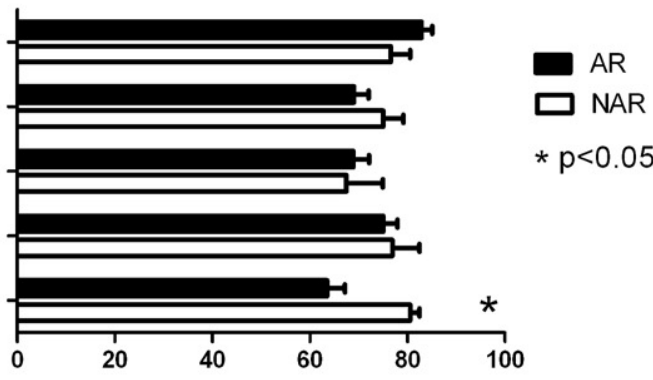

Cardiac Module Self-Report

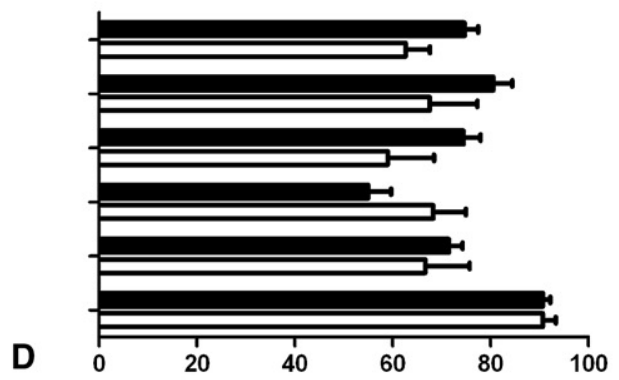

Quality of Life Score

FIGURE 1. Comparison of QOL scores between AR and NAR groups within individual functional domains on Peds QL Generic Scales (A, B) and Cardiac Module (C, D). Values are mean scores by group. Error bars represent 1 standard deviation. $* P<.05$. AR, Anatomic repair; NAR, non-anatomic repair. 

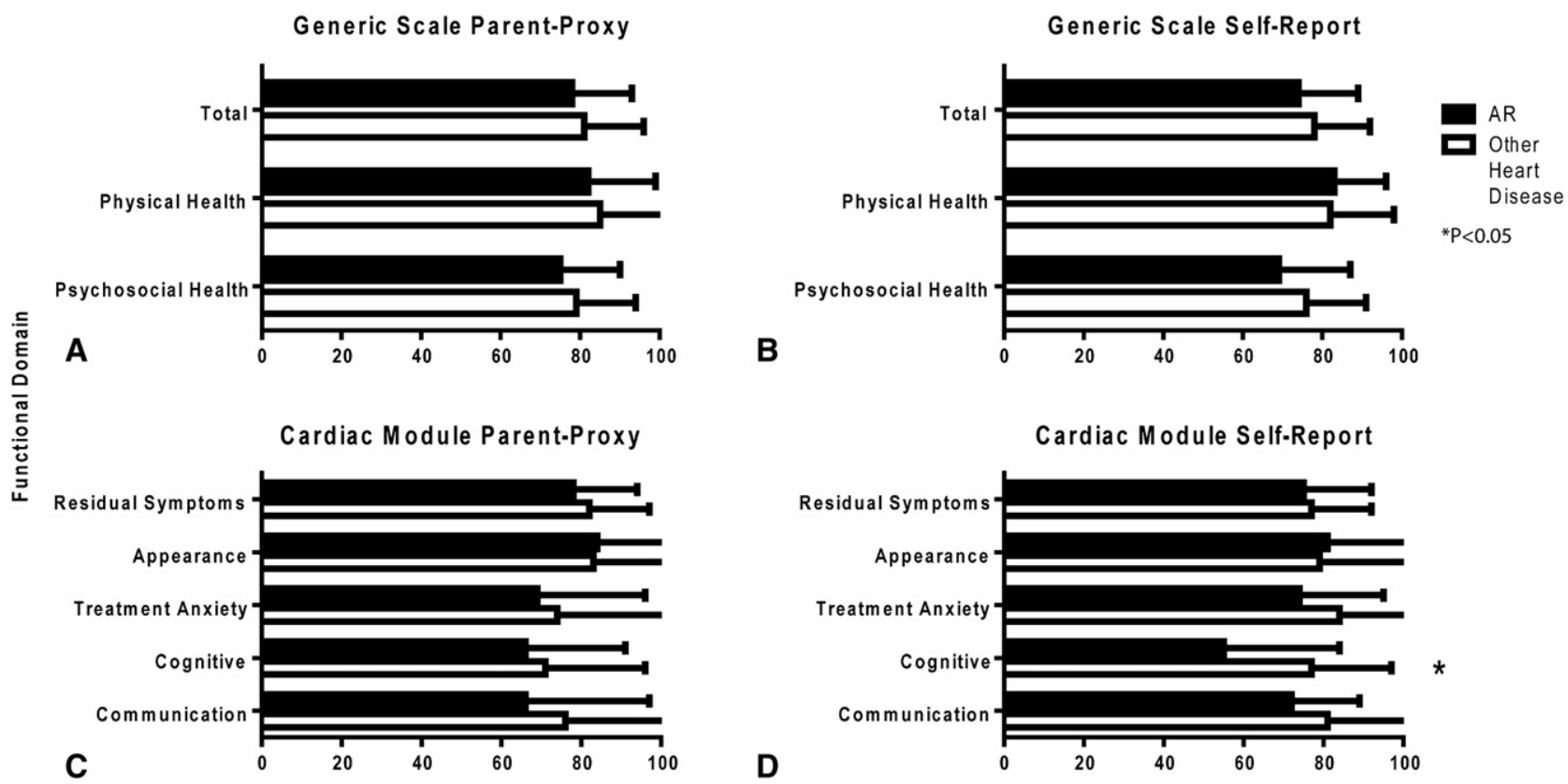

Quality of Life Score

FIGURE 2. Comparison of QOL scores between AR group and patients with moderate or severe heart disease (11) within individual functional domains on Peds QL Generic Scales (A, B) and Cardiac Module (C, D). Values are mean scores by group. Error bars represent 1 standard deviation. *P<.05. AR, Anatomic repair.

\section{Analysis of Clinical Risk Factors for Low Reported Quality of Life}

We focused our analysis of clinical variables affecting QOL after AR on the 3 risk factors described previously: (1) major in-hospital morbidity, (2) prolonged hospital stay, and (3) pacemaker dependence. The parent-proxy scores for both scales are shown in Figure 3. In-hospital morbidity was not associated with lower perceived QOL (Figure 2, A). In contrast, parent-proxies of patients who had a prolonged hospital stay $(\mathrm{n}=8)$ reported significantly lower QOL in the domain of residual symptoms compared with those who stayed less than 28 days after AR (Figure 2,B), and the point estimates in several other domains were lower for those with a prolonged hospital stay, although not statistically significant. Similarly, parent- proxies of patients with pacemakers $(n=9)$ reported lower QOL in all functional domains compared with patients without pacemakers (Figure 2, C), but patients with pacemakers did not self-report lower QOL (analysis not shown).

\section{DISCUSSION}

This study compared 2 management strategies (AR vs conventional or NAR) for patients with variants of CCTGA using perceived health-related QOL as the primary outcome measure. To our knowledge, this is the first time that QOL has been used as the primary outcome to compare different surgical management strategies in children with CHD.

The results suggest that patients who have undergone AR and their parent-proxies report similar QOL to those in the NAR group. One area of difference was in the domain of
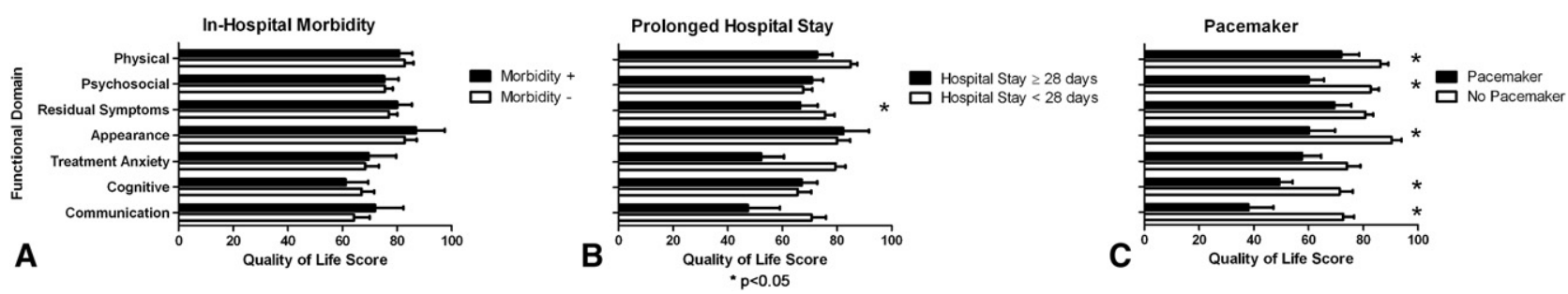

FIGURE 3. Comparison of parent-proxy QOL scores between patients exposed and not exposed to a priori defined risk factors for lower reported QOL. Risk factors include major in-hospital morbidity (A), prolonged hospital stay (B), and need for permanent pacemaker (C). Scores from both the Peds QL Generic Scales and Cardiac Module are reported (subcomponents of the psychosocial composite domain are not displayed). Black bars represent mean scores of patients exposed to the risk factor. Error bars represent 1 standard deviation. $* P<.05$. 
school functioning, where the AR group self-reported a significantly lower score. Parents of patients in the AR group did not rate school performance lower than parents of patients in the NAR group, and they gave a higher score relative to self-reports in the AR group. There was also a consistent pattern of lower reported functioning by both groups in the domains of cognition and communication. Children with complex CHD requiring surgery are at risk for lower school performance, ${ }^{13-15}$ but it is not known whether perceived QOL in the domains of school function, communication, and cognition correlate with objective performance measures. Specific exposures in the perioperative course after AR may place children at risk for later cognitive difficulties, but these are as yet unknown. Future studies of patients with CCTGA and other forms of complex CHD are necessary to determine whether perceived deficiencies in school functioning are common, the reasons for a discrepancy between perceptions of children and parents, and whether the perception of the children matches objective measures of school performance.

The relative risks of the more complex AR strategy must be weighed against the known risks of right ventricular failure and late mortality after conventional repair or medical management. The theoretic advantages of AR may be expected to have a greater positive impact on patients' perceived QOL (relative to the NAR group) as they enter adulthood, when the morbidity associated with systemic right ventricular failure is likely to manifest. However, there is an equally valid counterargument: Given the expected late complications of atrial switch procedures, ${ }^{16,17}$ including sinus node dysfunction and arrhythmias, and the limited reports of left ventricular dysfunction ${ }^{18,19}$ and neoaortic insufficiency $^{6,18}$ after AR, there exists the possibility that patients who have undergone AR will experience morbidities that negatively affect their perceived QOL as they enter adulthood. These uncertainties underscore the need for repeated assessment of similar cohorts to determine how QOL changes over time in patients with this disease.

AR has been our preferred operative strategy for patients with CCTGA since 1993. As such, the patients in the NAR group were essentially historical controls; subjects in the NAR group were older and had their operations several years before those in the AR group. Thus, our NAR group may not be representative of patients who would be treated similarly in the current era. We performed an analysis comparing the AR group with a modern cohort of patients with mixed diagnoses rated as moderate to severe, some of whom had surgery and were with or without residual cardiac disease. The working assumption was that this cohort approximates what would be expected from conventional repair or medical management of CCTGA in the current era with regard to disease complexity. Patients in the AR group generally rated their QOL similarly to the mixed co- hort, but consistently reported lower scores in the domains of cognition and communication. A direct comparison between patients undergoing AR and patients with CCTGA who have a systemic right ventricle managed in the modern era is needed to determine if one strategy produces better QOL outcomes.

Our analysis of clinical risk factors and their influence on perceived QOL in patients who have undergone AR demonstrated that a prolonged hospital stay was associated with lower QOL in several functional domains consistent with previous reports for other critically ill children. ${ }^{20,21}$ Although this may be expected to be due to greater morbidity experienced by the group with prolonged hospital length of stay, we did not find an association between major in-hospital morbidity and lower perceived QOL. It is possible that patients in the AR group who had a prolonged hospitalization have chronic problems that are directly associated with responses on the Peds QL. Further evaluation of patients' late physiologic status, need for reintervention, and chronic morbidities as risk factors for lower QOL after AR will be necessary to delineate these associations. It is also possible that prolonged hospitalization affects perceived QOL independently, and enhancing our understanding of this effect may help guide the postoperative care of patients after AR and other complex congenital heart surgery.

In this cohort, parents of patients with pacemakers perceived a significantly lower QOL for their children compared with parents of patients without pacemakers. However, pacemaker-dependent patients did not selfreport lower QOL. Limited data exist on the QOL impact of permanent pacing in children and young adults. Limited data suggest that permanent pacing is associated with lower QOL in adult patients undergoing tricuspid valve operations. ${ }^{22}$ Understanding the relationship between pacemaker dependence and QOL is important in anticipating how perceived QOL may change over time in patients who have undergone AR. The combined risks of complete heart block in this disease with the risk of tachyarrhythmia after atrial switch operations make it possible that a significant number of adult survivors of AR will require pacemaker therapy. If pacemaker dependence continues to be a significant risk factor for lower QOL in future studies, this may factor into the surgical decision-making for patients, especially those at higher risk of perioperative heart block.

This investigation has some important limitations. This study was conducted within a relatively small cohort from a single institution; these were convenience samples that were not designed to achieve adequate statistical power to test specific null hypotheses. As such, the presence or lack of statistically significant differences between groups should be interpreted with appropriate caution. The groups were not randomized to one management strategy; there were patients in the AR group who had previously failed 
NAR strategies. In addition, patients in the NAR group were older, and changes in surgical and postoperative care over time are potential confounding variables. Follow-up data on physiologic status (ie, ventricular function, residual cardiac disease), exercise capacity, neurodevelopment, and objective measures of school performance were not available to us to analyze in conjunction with perceived QOL responses. The majority of this cohort is followed at other institutions, making the regulatory and logistic obstacles to collecting these data prohibitive. Future analyses would benefit from the inclusion of these data to more completely understand the determinants of QOL. We did not record a measure of the socioeconomic status of respondents, which may also affect perceived QOL.

\section{CONCLUSIONS}

We have performed an assessment of 2 management strategies for patients with CCTGA using perceived QOL as the primary outcome measure. At intermediate followup, patients who have undergone AR at our institution report similar QOL to patients who have a systemic right ventricle. Perceived QOL will be important in the ongoing multifactorial assessment of the risks and benefits of the technically feasible, yet complicated, AR strategy. Further research is necessary to determine the physiologic and functional markers that correlate with patients' perceived QOL. Multi-institutional collaboration will be imperative to generate cohorts sufficient in size to answer questions related to this rare disease.

\section{References}

1. Devaney EJ, Charpie JR, Ohye RG, Bove EL. Combined arterial switch and Senning operation for congenitally corrected transposition of the great arteries: patient selection and intermediate results. J Thorac Cardiovasc Surg. 2003;125: 500-7.

2. Duncan BW, Mee RB, Mesia CI, Qureshi A, Rosenthal GL, Seshadri SG, et al. Results of the double switch operation for congenitally corrected transposition of the great arteries. Eur J Cardiothorac Surg. 2003;24:11-20.

3. Ilbawi MN, Ocampo CB, Allen BS, Barth MJ, Roberson DA, Chiemmongkoltip P, et al. Intermediate results of the anatomic repair for congenitally corrected transposition. Ann Thorac Surg. 2002;73:594-600.

4. Koh M, Yagihara T, Uemura H, Kagisaki K, Hagino I, Ishizaka T, et al. Intermediate results of the double-switch operations for atrioventricular discordance. Ann Thorac Surg. 2006;81:671-7.

5. Langley SM, Winlaw DS, Stumper O, Dhillon R, De Giovanni JV, Wright JG, et al. Midterm results after restoration of the morphologically left ventricle to the systemic circulation in patients with congenitally corrected transposition of the great arteries. J Thorac Cardiovasc Surg. 2003;125:1229-41.
6. Sharma R, Talwar S, Marwah A, Shah S, Maheshwari S, Suresh P, et al. Anatomic repair for congenitally corrected transposition of the great arteries. $J$ Thorac Cardiovasc Surg. 2009; 137:404-e4.

7. Shin'oka T, Kurosawa H, Imai Y, Aoki M, Ishiyama M, Sakamoto T, et al. Outcomes of definitive surgical repair for congenitally corrected transposition of the great arteries or double outlet right ventricle with discordant atrioventricular connections: risk analyses in 189 patients. J Thorac Cardiovasc Surg. 2007;133: 1318-28, 28 e1-e4.

8. Graham TP Jr, Bernard YD, Mellen BG, Celermajer D, Baumgartner H, Cetta F, et al. Long-term outcome in congenitally corrected transposition of the great arteries: a multi-institutional study. J Am Coll Cardiol. 2000;36:255-61.

9. Varni JW, Burwinkle TM, Seid M, Skarr D. The PedsQL 4.0 as a pediatric population health measure: feasibility, reliability, and validity. Ambul Pediatr. 2003; 3:329-41.

10. Uzark K, Jones K, Burwinkle TM, et al. The Pediatric Quality of Life Inventory in children with heart disease. Prog Pediatr Cardiol. 2003;18:141-8.

11. Uzark K, Jones K, Slusher J, Limbers CA, Burwinkle TM, Varni JW. Quality of life in children with heart disease as perceived by children and parents. Pediatrics. 2008;121:e1060-7.

12. Gaies MG, Goldberg CS, Ohye RG, Devaney EJ, Hirsch JC, Bove EL. Early and intermediate outcome after anatomic repair of congenitally corrected transposition of the great arteries. Ann Thorac Surg. 2009;88:1952-60.

13. Mahle WT, Clancy RR, Moss EM, Gerdes M, Jobes DR, Wernovsky G. Neurodevelopmental outcome and lifestyle assessment in school-aged and adolescent children with hypoplastic left heart syndrome. Pediatrics. 2000;105:1082-9.

14. Shillingford AJ, Glanzman MM, Ittenbach RF, Clancy RR, Gaynor JW, Wernovsky G. Inattention, hyperactivity, and school performance in a population of school-age children with complex congenital heart disease. Pediatrics. 2008; 121:e759-67.

15. Granberg M, Rydberg A, Fisher AG. Activities in daily living and schoolwork task performance in children with complex congenital heart disease. Acta Paediatr. 2008;97:1270-4.

16. Bouchardy J, Therrien J, Pilote L, Ionescu-Ittu R, Martucci G, Bottega N, et al. Atrial arrhythmias in adults with congenital heart disease. Circulation. 2009;120: 1679-86.

17. Kammeraad JA, van Deurzen CH, Sreeram N, Bink-Boelkens MT, Ottenkamp J, Helbing WA, et al. Predictors of sudden cardiac death after Mustard or Senning repair for transposition of the great arteries. J Am Coll Cardiol. 2004;44: 1095-102.

18. Bautista-Hernandez V, Marx GR, Gauvreau K, Mayer JE Jr, Cecchin F, del Nido PJ. Determinants of left ventricular dysfunction after anatomic repair of congenitally corrected transposition of the great arteries. Ann Thorac Surg. 2006;82:2059-66.

19. Quinn DW, McGuirk SP, Metha C, Nightingale P, de Giovanni JV, Dhillon R, et al. The morphologic left ventricle that requires training by means of pulmonary artery banding before the double-switch procedure for congenitally corrected transposition of the great arteries is at risk of late dysfunction. J Thorac Cardiovasc Surg. 2008;135:1137-44, 44 e1-e2.

20. Conlon NP, Breatnach C, O'Hare BP, Mannion DW, Lyons BJ. Health-related quality of life after prolonged pediatric intensive care unit stay. Pediatr Crit Care Med. 2009;10:41-4.

21. Buysse CM, Raat H, Hazelzet JA, Hop WC, Maliepaard M, Joosten KF. Surviving meningococcal septic shock: health consequences and quality of life in children and their parents up to 2 years after pediatric intensive care unit discharge. Crit Care Med. 2008;36:596-602.

22. Jokinen JJ, Turpeinen AK, Pitkanen O, Hippelainen MJ, Hartikainen JE. Pacemaker therapy after tricuspid valve operations: implications on mortality, morbidity, and quality of life. Ann Thorac Surg. 2009;87:1806-14. 